\title{
Deconfinement and the Hagedorn Transition in String Theory
}

\author{
Shyamoli Chaudhuri f \\ 214 North Allegheny St. \\ Bellefonte, PA 16823
}

Abstract
Superseded and extended in hep-th/0105110 and hep-th/0208112.

${ }^{1}$ shyamoli@thphysed.org 


\section{Introduction}

This paper has been superseded by hep-th/0105110 and hep-th/0208112. The results in the published letter are not incorrect, but the original presentation has been extensively rewritten to clarify that thermal duality relations arise naturally as a consequence of modular invariance in closed

string gases. Corresponding results for the type I open and closed string gas are included in hepth/0208112 giving a more unified picture of the statistical mechanics of fermionic string gases, and of the phase transition to the long string phase. The pedagogical case of the free closed bosonic string gas appears in hep-th/0105110. 